\title{
RADIO ECHO SOUNDING OF HORIZONTAL LAYERS IN ICE
}

\author{
By C. H. Harrison \\ (Scott Polar Research Institute, Cambridge, England)
}

\begin{abstract}
Radio echo-sounding surveys of Antarctica and Greenland have revealed extensive layering within the ice. Formulae for the effective reflection coefficient, when viewed by a pulsed radar, are derived for isolated or multiple randomly spaced layers. In the latter case the variation in dielectric constant with depth is described by a vertical autocorrelation function and a standard deviation. Some measurements of the reflection coefficient of layers, and the dielectric absorption of ice are given. The significance of the fading of layer echoes and the possible causes of variations in the dielectric constant are considered and some further investigations are suggested.
\end{abstract}

It is concluded that the echo strengths found in the Antarctic may be explained by multiple layering, and that the necessary fractional change in the dielectric constant may be as small as $10^{-4}$. It is suggested that this change in dielectric constant may be due to differences in orientation of anisotropic ice crystals.

RÉsumÉ. Sondages de niveaux horizontaux dans la glace par echo-radio. Une campagne de sondages par échoradio sur l'Antarctique et le Groenland ont révélé une stratification horizontale très poussée à l'intérieur de la glace. Les formules pour le coefficient effectif de réflexion, lors du survol par un radar pulsé, sont établies pour un niveau isolé ou pour des niveaux multiples distribués au hasard. Dans ce dernier cas, la variation de la constante diélectrique avec la profoundeur est représentée par une fonction d'autocorrélation verticale et une déviation standard. On donne quelques mesures de coefficients de réflexion de certains niveaux, ainsi que l'absorbtion diélectrique de la glace. On étudie la signification de l'évanouissement des échos de certains niveaux, ainsi que les causes possibles des variations de la constante diélectrique, on en tire quelques suggestions pour de nouvelles recherches.

On conclut que la puissance des échos obtenus dans l'Antarctique peut s'expliquer par la présence de niveaux multiples et que la variation relative qu'il est nécessaite d'admettre pour la constante diélectrique peut être très faible, de l'ordre de $1 \mathrm{O}^{-4}$. On émet l'hypothèse que cette variation de la constante diélectrique peut être due à des différences dans l'orientation de cristaux anisotropiques de glace.

Zusammenfassung. Radio-Echolotungen horizontaler Schichten in Eis. Radarseismische Messungen in der Antarktis und in Grönland erwiesen eine ausgedehnte Schichtung im Eis. Formeln für den effektiven Reflexionskoeffizienten eines gepulsten Radars bei isolierten oder mehrfachen, zufällig verteilten Schichten werden hergeleitet. Im zweiten Fall lässt sich die Änderung der Dielektrizitätskonstante durch eine vertikale Autokorrelationsfunktion und eine Standardabweichung beschreiben. Einige Messungen des Reflexionskoeffizienten an Schichten und der dielektrischen Absorption von Eis werden angegeben. Die Bedeutung des Verlöschens von Schichtechos und die möglichen Ursachen von Änderungen der Dielektrizitätskonstante werden betrachtet und einige weitere Untersuchunen werden vorgeschlagen.

Es lässt sich schliessen, dass Echostärken, wie sie in der Antarktis auftreten, auf mehrfache Schichten zurückzuführen sind und dass die dafür notwendige relative Änderung der Dielektrizitätskonstante nicht grösser als $\mathrm{IO}^{-4} \mathrm{zu}$ sein braucht. Als Erklärung für diesen Wechsel der Dielektrizitätskonstante werden Unterschiede in der Orientierung anisotroper Eiskristalle vorgeschlagen.

\section{INTRODUCTION}

Airborne radio echo surveying has been used principally as a technique for depth sounding the polar ice sheets, but in the deep inland ice of Greenland and the Antarctic partial reflections from intermediate layers are common (Robin and others, I970, Gudmandsen 1970). The layer echoes have been of glaciological interest in demonstrating the deformation of ice over large bedrock undulations, but the nature of the layers themselves, as deduced from radio echo data, is also glaciologically significant.

The radio echo-sounding apparatus referred to in this paper is an airborne pulsed radar operating at $35 \mathrm{MHz}$ (and more recently at $60 \mathrm{MHz}$ ), with an aerial beam width broad along the line of flight and narrow across it, recording echo delays continuously as a range profile on $35 \mathrm{~mm}$ photographic film (Z-scope display). Details of the system design are given in Evans and Smith (1969), and some of the interpretation problems resulting from the broad beam width are discussed in Harrison (1970, 1971).

Three large-scale airborne surveys in the Antarctic were carried out between I969 and I97I by the Scott Polar Research Institute with logistic support from the U.S. National Science Foundation (Evans and others, 1972). These will be referred to as S.P.R.I. surveys. Figure I shows the rough geographical distribution of layer echoes observed on these flights. 


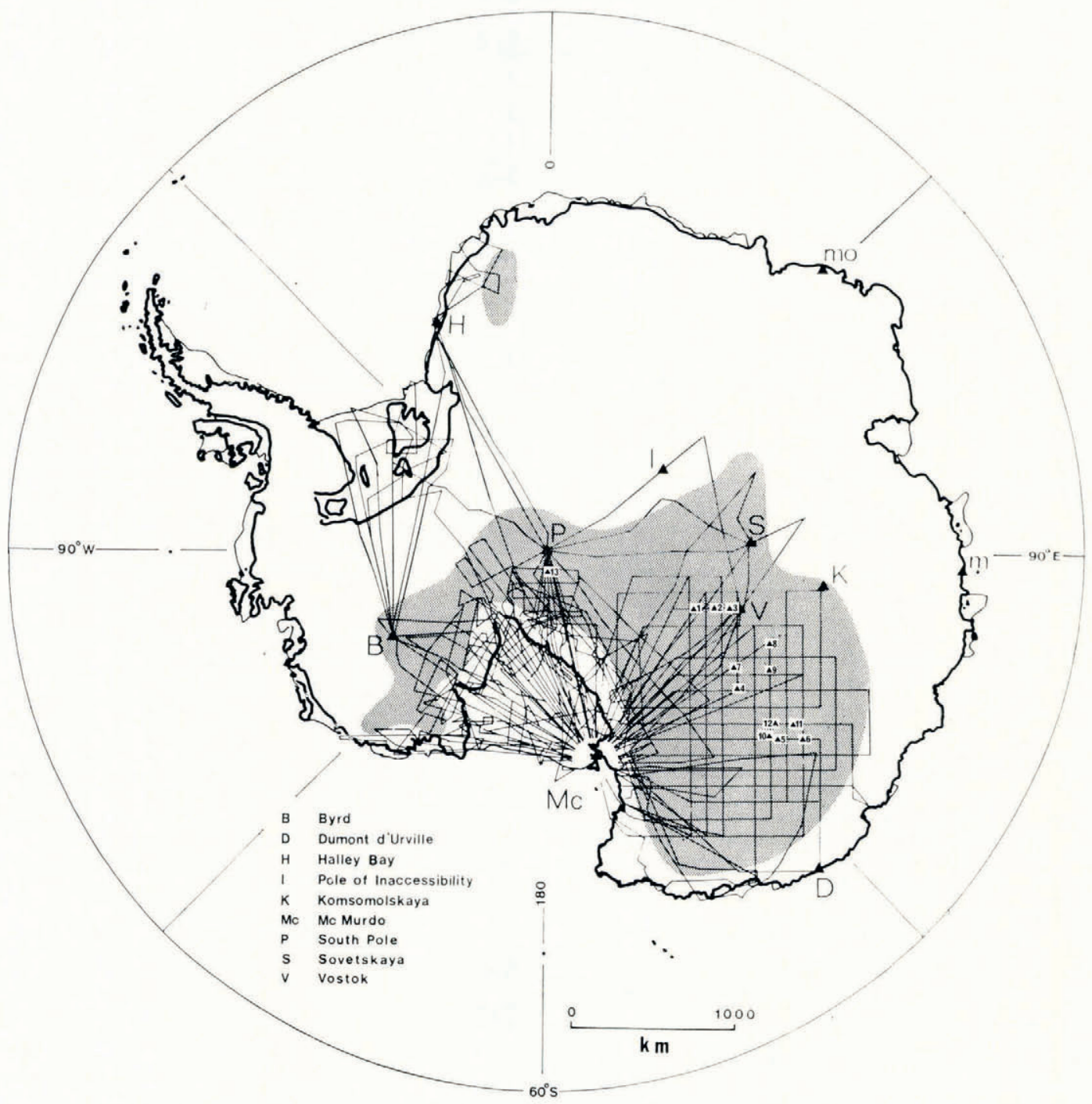

Fig. I. The fight lines covered by the S.P.R.I.-N.S.F. surveys in the Antarctic and the geographical distribution of observed layer echoes (stipple). Measurements of the reflection coefficient of the layers and the attenuation per unit depth have been made at the numbered sites (Table I).

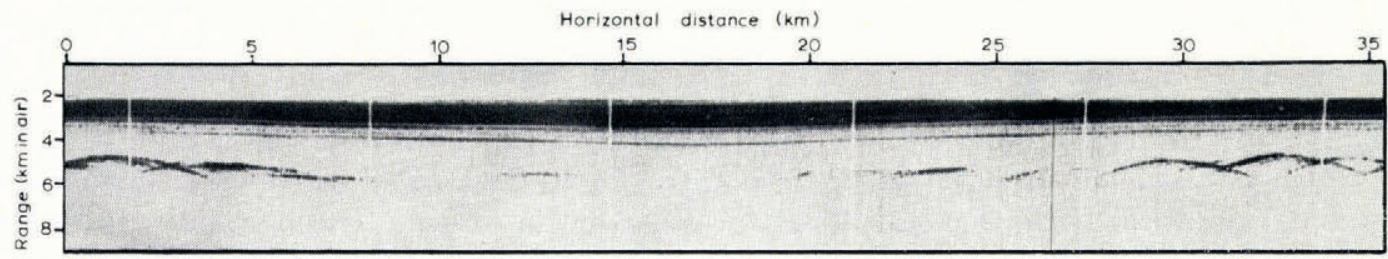

Fig. 2. Extensive layer echoes. 
They were seen near Byrd Station and on the Antarctic Plateau, but so far they have not been seen on ice shelves, possibly because of instrumental problems during the 1969-70 season in which most of the ice-shelf flying was done. Individual echoes can often be traced for $5^{\circ}$ to $100 \mathrm{~km}$ along a flight line sometimes following bedrock relief as shown in Figure 2, and they usually have a low spatial fading rate as in Figure 3 which suggests that they come from smooth reflectors. However, layers at spacings less than about half a pulse length cannot be resolved by the echo sounder, so a continuous echo on the record does not necessarily represent a single layer.

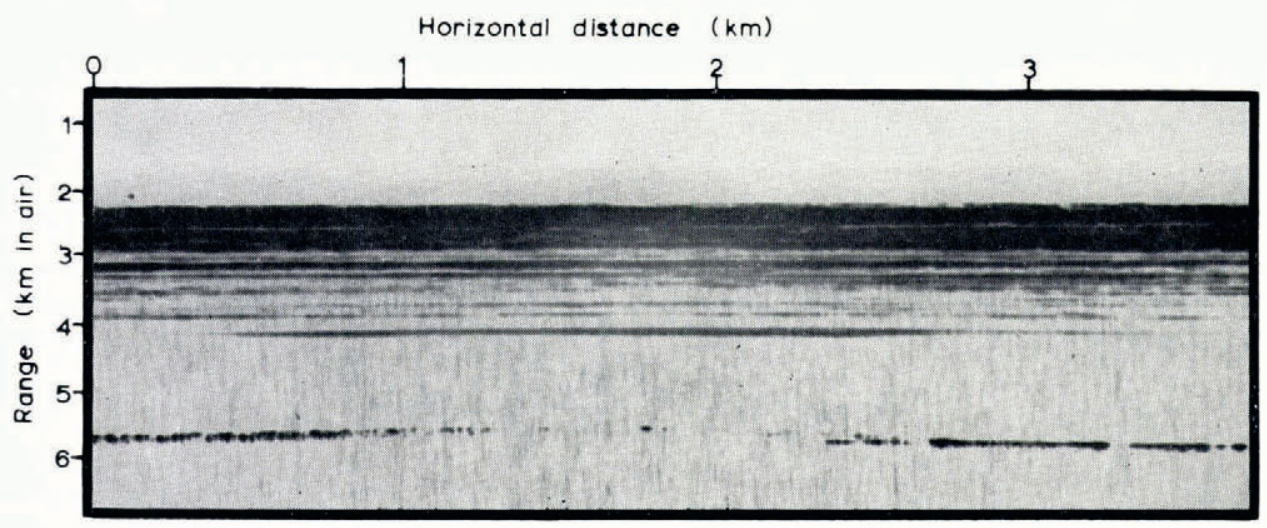

Fig. 3. Layer echoes showing the characteristic low fading rates (long fading length) and the characteristic vertical spacing.

Treating the reflections as alternate layers of bubbly and clear ice, Robin and others ( I969) showed that a single layer about $0 . \mathrm{I} \mathrm{m}$ thick with a difference in density of $0.0 \mathrm{I}$ $\mathrm{Mg} \mathrm{m} \mathrm{m}^{-3}$ could account for an observed reflection coefficient of $-70 \mathrm{~dB}$. However, Gudmandsen (1970) and S.P.R.I. have since obtained layer echoes from depths greater than $2500 \mathrm{~m}$ where, according to Schytt (I958), density variations should be very much smaller than o.or $\mathrm{Mg} \mathrm{m}^{-3}$, so the effective reflection coefficients must arise from some other mechanism as well. This will be considered in more detail later.

\section{THE POWER REFLECTED BY LAYERS}

For the purposes of calculating the power reflected from layers we begin by assuming that the ice is horizontally stratified and that the dielectric constant at any point is given by $\epsilon=\epsilon_{\mathrm{I}}+\Delta \epsilon(z)$ where $\epsilon_{\mathrm{I}}$ is the mean and $\Delta \epsilon\left(\ll \epsilon_{\mathrm{I}}\right)$ is a function of depth only. Three models for the variation of $\Delta \epsilon$ with depth will be considered:

I. Isolated thin layers at spacings greater than a pulse length (Fig. 4(a));

2. A random variation with depth where $\Delta \epsilon$ is statistically stationary over the depth of the medium, in other words the average properties of $\Delta \epsilon$ remain the same throughout the medium (Fig. 4(b));

3. A random variation with depth where $\Delta \epsilon$ is statistically stationary over distances of the same order as the pulse length, in other words $\Delta \epsilon$ may change randomly on a scale smaller than the pulse length, but the average properties of $\Delta \epsilon$ may change smoothly over distances larger than the pulse length (Fig. 4(c)).

Irregularities of large horizontal extent and small vertical extent (compared with the radio wavelength) reflect specularly, and so it is convenient to treat layers, or groups of layers, as one specular reflector and to calculate the effective power reflection coefficient $R$. We may obtain a general formula for the amplitude reflection coefficient $R_{\mathrm{A}}$ by comparing the Kirchhoff 


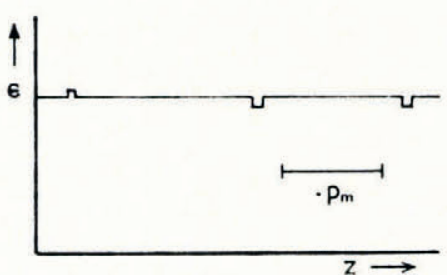

(a)

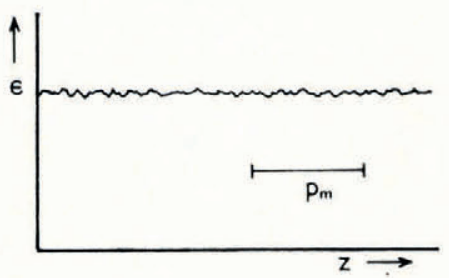

(b)

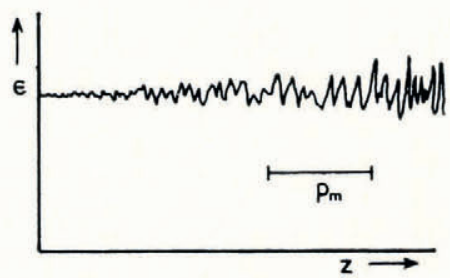

(C)

Fig. 4. Three hypothetical variations of $\epsilon$ with depth showing the relative magnitude of the pulse length $p_{\mathrm{m}}:(a)$ isolated thin layers, (b) random variations of $\Delta \epsilon$, and (c) a random variation of $\Delta \epsilon$ where the average properties may change over a distance greater than the pulse length.

approximation for the field reflected by an element of a plane reflector with the field reradiated by a small element of volume with dielectric constant $\epsilon(z)=\epsilon_{\mathrm{I}}+\Delta \epsilon(z)$.

By the Kirchhoff approximation the electric field $E$ at the receiver at a distance $z_{0}$ from a plane reflector is

$$
E=\frac{\mathrm{i}}{\lambda_{\mathrm{m}}} E_{0} \frac{\exp \left(2 \mathrm{i} k_{\mathrm{m}} z_{0}\right)}{z_{0}} \int_{S} R_{\mathrm{A}} Q \mathrm{~d} S
$$

where $E_{0}$ is the strength of the field incident on the reflector, $\lambda_{\mathrm{m}}=2 \pi / k_{\mathrm{m}}$ is the wavelength in the medium, $\mathrm{d} S$ is an element of surface on the plane, and $Q$ is a function which describes the phase variations across the surface $S$ due to the curved incident wavefronts. The layered medium may be split up into elements of volume $\mathrm{d} V=\mathrm{d} z \cdot \mathrm{d} S$ much smaller than the irregularities where the dielectric constant deviates from the mean by $\Delta \epsilon$, and each volume $\mathrm{d} V$ becomes polarized by the incident field and re-radiates as an oscillating dipole (Booker and Gordon, 1950). Provided $\Delta \epsilon \ll \epsilon_{\mathrm{I}}$, the incident field is unaltered by the presence of local dipoles, and the electric field at a polar angle $\gamma$ and a distance $r$ from the oscillating elementary dipole is given by

$$
E=\frac{\pi \Delta \epsilon}{r \lambda_{\mathrm{m}}^{2} \epsilon} E_{0} \mathrm{~d} V \sin \gamma \exp \left(\mathrm{i} k_{\mathrm{m}} r\right) .
$$

The induced dipoles are parallel to the incident field $E_{0}$ and perpendicular to the incident ray path. Since we are interested only in back scattering, the induced dipoles are also perpendicular to the return ray path and $\operatorname{sos} \sin \gamma=\mathrm{I}$. Including the fact that $E_{0}$ is spatially limited by the pulse modulation, we have from Equation (2)

$$
E=\frac{\pi}{\lambda_{\mathrm{m}}^{2}} E_{0} \frac{\exp \left(2 \mathrm{i} k_{\mathrm{m}} z_{0}\right)}{z_{0}} \int_{S} \int_{z=-\infty}^{\infty} \frac{\Delta \epsilon(z)}{\epsilon_{\mathrm{I}}} \exp \left[2 \mathrm{i} k_{\mathrm{m}}\left(z-z_{0}\right)\right] \exp -\left[\frac{2\left(z-z_{0}\right)}{p_{\mathrm{m}}}\right]^{2} Q \mathrm{~d} z \mathrm{~d} S(3)
$$

where the pulse shape has been taken to be a Gaussian exp $-\left[\left(z-z_{0}\right) / p_{\mathrm{m}}\right]^{2}$ of half width $p_{\mathrm{m}}$ (thus $p_{\mathrm{m}}$ is approximately half a pulse length in the medium). Combining Equations ( $\mathrm{I}$ ) and (3) we have

$$
R_{\mathrm{A}}=-\frac{\mathrm{i} \pi}{\lambda_{\mathrm{m}}} \int_{z=-\infty}^{\infty} \frac{\Delta \epsilon(z)}{\epsilon_{\mathrm{I}}} \exp \left[2 \mathrm{i} k_{\mathrm{m}}\left(z-z_{0}\right)\right] \exp -\left[\frac{2\left(z-z_{0}\right)}{p_{\mathrm{m}}}\right]^{2} \mathrm{~d} z
$$

This general result may be used to calculate the effective power reflection coefficient for any given variation of $\epsilon$ with $z$. 
Case I. Isolated thin layer

Taking

$$
\begin{cases}\Delta \epsilon(z)=-\Delta \epsilon & \text { for } z_{0}<z<z_{0}+l, \\ \Delta \epsilon(z)=0 & \text { for } z<z_{0} \text { and } z>z_{0}+l,\end{cases}
$$

we have from Equation (4)

$$
\begin{aligned}
& R=\left|R_{\mathrm{A}}\right|^{2}=\frac{\pi^{2}}{\lambda_{\mathrm{m}^{2}}}\left(\frac{\Delta \epsilon}{\epsilon_{\mathrm{I}}}\right)^{2}\left|\int_{z=z_{0}}^{z_{0}+l} \exp \left[2 \mathrm{i} k_{\mathrm{m}}\left(z-z_{0}\right)\right] \mathrm{d} z\right|^{2}, \\
& R=\frac{1}{4}\left(\frac{\Delta \epsilon}{\epsilon_{\mathrm{I}}}\right)^{2} \sin ^{2} k_{\mathrm{m}} l .
\end{aligned}
$$

For small $k_{\mathrm{m}} l$ this is identical to the expression derived by Robin and others (1969, equation (16)) where a relation between $\epsilon$ and ice density was included in the result.

$$
R=\frac{\pi^{2} l^{2}}{\lambda_{\mathrm{m}^{2}}}\left(\frac{\Delta \epsilon}{\epsilon_{\mathrm{I}}}\right)^{2}
$$

\section{Case 2. Random variations}

Assuming that $\Delta \epsilon$ is statistically stationary over the depth of the medium (a distance much larger than a pulse length) we may describe the variation of $\Delta \epsilon$ with $z$ by an autocorrelation function $\rho_{\varepsilon}(\zeta)$, where

$$
\rho_{\epsilon}(\zeta)=\frac{\int_{-\infty}^{\infty} \Delta \epsilon(z) \Delta \epsilon(z+\zeta) \mathrm{d} z}{\int_{-\infty}^{\infty}[\Delta \epsilon(z)]^{2} \mathrm{~d} z}=\frac{\langle\Delta \epsilon(z) \Delta \epsilon(z+\zeta)\rangle}{\left\langle\Delta \epsilon(z)^{2}\right\rangle}
$$

and a convenient and plausible function is a Gaussian

$$
\rho_{\varepsilon}(\zeta)=\exp \left(-\frac{\zeta^{2}}{l^{2}}\right)
$$

where $l$ is now the correlation length of $\Delta \epsilon$ or the characteristic spacing of layers. We can calculate the mean power reflection coefficient from

$$
R=R_{\mathrm{A}}\left(z_{0}\right) R_{\mathrm{A}} \star\left(z_{0}\right)
$$

and we may also estimate the characteristic width or spacing of the layers as seen on the radio echo record. This is given by the width of the autocorrelation function of received power in range space $\rho_{\mathrm{P}}(\tau)$, and from Ratcliffe (1956, p. 232), when the components of back-scattered complex amplitude have completely random phase, we have

$$
\rho_{\mathrm{P}}(\tau)=\left|\rho_{\mathrm{A}}(\tau)\right|^{2}
$$

where $\rho_{\mathrm{A}}(\tau)$ is the autocorrelation function of received amplitude given by

$$
\rho_{\mathrm{A}}(\tau)=\frac{\int_{-\infty}^{\infty} R_{\mathrm{A}}\left(z_{0}\right) R_{\mathrm{A}} \star\left(z_{0}+\tau\right) \mathrm{d} z_{0}}{\int_{-\infty}^{\infty} R_{\mathrm{A}}\left(z_{0}\right) R_{\mathrm{A}} \star\left(z_{0}\right) \mathrm{d} z_{0}} .
$$


Using Equations (4) and (10) we have

$$
\begin{aligned}
& R=\frac{\pi^{2}}{\lambda_{\mathrm{m}}^{2}} \int_{-\infty}^{\infty} \int_{-\infty}^{\infty} \frac{\Delta \epsilon(z)}{\epsilon} \frac{\Delta \epsilon\left(z^{\prime}\right)}{\epsilon} \exp 2 \mathrm{i} k_{\mathrm{m}}\left[\left(z-z_{0}\right)-\left(z^{\prime}-z_{0}\right)\right] \times \\
& \times \exp -\left\{\left[\frac{2\left(z-z_{0}\right)}{p_{\mathrm{m}}}\right]^{2}+\left[\frac{2\left(z^{\prime}-z_{0}\right)}{p_{\mathrm{m}}}\right]^{2}\right\} \mathrm{d} z \mathrm{~d} z^{\prime},
\end{aligned}
$$

and changing variables from $z, z^{\prime}$ to $z$, $\zeta$ where $z^{\prime}=z-\zeta$,

$$
\begin{aligned}
R=\frac{\pi^{2}}{\lambda_{\mathrm{m}}^{2}}\left\langle\left(\frac{\Delta \epsilon}{\epsilon}\right)^{2}\right\rangle \int_{-\infty}^{\infty} \int_{-\infty}^{\infty} \rho_{\epsilon}(\zeta) \exp \left(2 \mathrm{i} k_{\mathrm{m}} \zeta\right) \times \\
\quad \times \exp -\left\{\left(\frac{2}{p_{\mathrm{m}}}\right)^{2}\left[2\left(z-z_{0}\right)^{2}-2\left(z-z_{0}\right) \zeta+\zeta^{2}\right]\right\} \mathrm{d} z \mathrm{~d} \zeta .
\end{aligned}
$$

Integrating with respect to $z$ and substituting Equation (9) in Equation (13)

$$
R=\frac{\pi^{2}}{\lambda_{\mathrm{m}^{2}}}\left\langle\left(\frac{\Delta \epsilon}{\epsilon}\right)^{2}\right\rangle p_{\mathrm{m}} \sqrt{\frac{\pi}{8}} \int_{-\infty}^{\infty} \exp -\left[\left(\frac{\mathrm{I}}{l^{2}}+\frac{2}{p_{\mathrm{m}^{2}}}\right) \zeta^{2}\right] \exp \left(2 \mathrm{i} k_{\mathrm{m}} \zeta\right) \mathrm{d} \zeta .
$$

Integrating with respect to $\zeta$ we have

$$
R=\frac{\pi^{3} p_{\mathrm{m}}}{\sqrt{ } 8 \lambda_{\mathrm{m}^{2}}\left(\frac{\mathrm{I}}{l^{2}}+\frac{2}{p_{\mathrm{m}^{2}}}\right)^{\frac{1}{2}}}\left\langle\left(\frac{\Delta \epsilon}{\epsilon}\right)^{2}\right\rangle \exp -\left[\frac{k_{\mathrm{m}^{2}}}{\left(\frac{\mathrm{I}}{l^{2}}+\frac{2}{p_{\mathrm{m}^{2}}}\right)}\right] .
$$

By a very similar method starting from Equation (12) we have

$$
\rho_{\mathrm{A}}(\tau)=\exp -\left[\frac{\tau^{2}}{\left(p_{\mathrm{m}}^{2} / 2+l^{2}\right)}\right] \exp \left[\frac{2 \mathrm{i} k_{\mathrm{m}} \tau}{\left(\mathrm{I}+2 l^{2} / p_{\mathrm{m}}^{2}\right)}\right] .
$$

The apparent spacing or width of the layers is, from Equations (II) and (I6), of the order of half a pulse length $p_{\mathrm{m}}$ or the real spacing of layers $l$, whichever is the larger. The term $\left[\left(\mathrm{I} / l^{2}\right)+\left(2 / p_{\mathrm{m}}^{2}\right)\right]^{\frac{1}{2}}$ in the mean power reflection coefficient given by Equation ( $\left.\mathrm{I}_{5}\right)$ becomes $\mathrm{I} / l$ for $l \ll p_{\mathrm{m}}$ and $\sqrt{ } 2 / p_{\mathrm{m}}$ for $l \gg p_{\mathrm{m}}$. However, in the latter case the reflection coefficient is $R \approx\left\langle\Delta \epsilon^{2} / \epsilon^{2}\right\rangle\left(k_{\mathrm{m}} p_{\mathrm{m}}\right)^{2} \exp -\frac{1}{2} k_{\mathrm{m}^{2}} p_{\mathrm{m}^{2}}$ and if the pulse contains ten radio-frequency cycles as is the case in the S.P.R.I. surveys, then $k_{\mathrm{m}} p_{\mathrm{m}} \approx 30$, so $R \approx\left\langle\Delta \epsilon^{2} / \epsilon^{2}\right\rangle \mathrm{IO}^{-}$. $^{200}$

As reflections of this strength are far too weak to be detected with the present apparatus we may restrict the treatment to the case where $l \ll p_{\mathrm{m}}$, so the width or spacing of detectable layers on the echo record is always of order $p_{\mathrm{m}}$, and the reflection coefficient is given by

$$
R=\frac{\pi^{3} p_{\mathrm{m}} l}{\sqrt{ } 8 \lambda_{\mathrm{m}^{2}}}\left\langle\left(\frac{\Delta \epsilon}{\epsilon}\right)^{2}\right\rangle \exp \left(-k_{\mathrm{m}^{2}}^{2}\right)
$$

Note that for $k_{\mathrm{m}} l \ll \mathrm{I}$ the reflection coefficient for multiple layering, Equation ( 17 ) is larger than that for a single layer, Equation (7) by a factor of approximately $p_{\mathrm{m}} / l$, the number of layers in a pulse length. Figure 5 shows the variation of $R$ with $k_{\mathrm{m}} l$ for a single layer, Equation (6), and multiple layers, Equation (1 7). For small $k_{\mathrm{m}} l, R$ increases in proportion to $l^{2}$ for a single layer but in proportion to $p_{\mathrm{m}} l$ for multiple layers. For large $k_{\mathrm{m}} l$ and multiple layers, Equation ( $\mathrm{I} 7), R$ decreases very rapidly beyond $k_{\mathrm{m}} l \approx 2$. There is a maximum value of $R$ for multiple layers occurring when $k_{\mathrm{m}} l=\mathrm{I} / \sqrt{ } 2,\left(l=\lambda_{\mathrm{m}} / 2 \sqrt{ } 2 \pi\right)$, and

$$
R_{\max }=\frac{\pi}{\mathrm{I} 6} p_{\mathrm{m}} k_{\mathrm{m}}\left\langle\left(\frac{\Delta \epsilon}{\epsilon}\right)^{2}\right\rangle \mathrm{e}^{-\frac{1}{2}}=\frac{\pi^{2}}{8} \frac{p_{\mathrm{m}} \mathrm{e}^{-\frac{1}{2}}}{\lambda_{\mathrm{m}}}\left\langle\left(\frac{\Delta \epsilon}{\epsilon}\right)^{2}\right\rangle .
$$


Remember that these formulae are for mean reflection coefficients taken over the many possible arrangements of $\Delta \epsilon(z)$ having the same statistical properties $\left\langle\Delta \epsilon^{2}\right\rangle$ and $\rho_{\varepsilon}(\zeta)$. Physically they correspond to the average of the reflection coefficients measured at a fixed depth but many widely spaced observer positions (or, in case 2, to the average over all depths at a fixed observer position).

It is interesting to note that on substituting $\lambda_{\mathrm{m}}=4.8 \mathrm{~m}, p_{\mathrm{m}}=22 \mathrm{~m}$, as for the S.P.R.I. apparatus, then to obtain a power reflection coefficient of ${ }_{10}^{-7}(-70 \mathrm{~dB})$ with a single layer, Equation (7), I cm thick we would need $\Delta \epsilon / \epsilon=5 \times \mathrm{IO}^{-2}$; to give the same reflection coefficient with multiple layering, Equation (I7), where $l=\mathrm{I} \mathrm{cm}$ we would need $\Delta \epsilon / \epsilon=\mathrm{I} \times \mathrm{IO}^{-3}$, or if $l \approx \lambda_{\mathrm{m}} / 2 \sqrt{ } 2 \pi$ giving the maximum reflection coefficient as in Equation (18), we would need only $\Delta \epsilon / \epsilon=1.7 \times 10^{-4}$. So it is clear that the echo sounder is very sensitive to variations of $\Delta \epsilon$ on a vertical scale of about $\lambda_{\mathrm{m}} / 2 \sqrt{ } 2 \pi$, which for $\lambda_{\mathrm{m}}=4.8 \mathrm{~m}$ is about $0.5 \mathrm{~m}$.

\section{Case 3. Variations of $R$ with depth}

We may treat smooth changes in the average properties of $\Delta \epsilon$ with depth as variations in $R$ with depth provided the changes are small over a pulse length. A gradual change in $R$ with depth may be effected by slow changes of $\left\langle\Delta \epsilon^{2}\right\rangle$ or $l$. The dependence of layer thickness $(l)$ on depth in an ice sheet has been calculated by Nye ( 1963$)$. On the assumption that there is negligible bottom melting and that the vertical plastic strain-rate along any vertical line is uniform at any given instant, he derives an expression for the thickness of a layer $l$ at height $h$ above the bottom of the ice.

$$
\frac{l}{L}=\frac{h}{H}
$$

where $L$ is the initial thickness of the layer and $H$ is the height above the bed at which the layer was formed, and all lengths are measured in ice equivalent so as not to include the effects of snow and firn compaction.

The variation of $\left\langle\Delta \epsilon^{2} / \epsilon^{2}\right\rangle$ with depth depends on the mechanism responsible for the changes of dielectric constant, and this will be considered in Section 4. If, for instance, the dielectric constant were dependent on density variations, $\left\langle\Delta \epsilon^{2} / \epsilon^{2}\right\rangle$ would decrease with depth, but if ash layers caused variations of dielectric constant, $\left\langle\Delta \epsilon^{2} / \epsilon^{2}\right\rangle$ might be nearly independent of depth. If, for lack of detailed information, we assume $\left\langle\Delta \epsilon^{2} / \epsilon^{2}\right\rangle$ is independent of depth, we may use Equation (I9) to substitute for $l$ in the expression for the power reflection coefficient. Knowing $L$ and $H$ we can find the dependence of $R$ on $h$ from Figure 5 . Taking $H$ as the
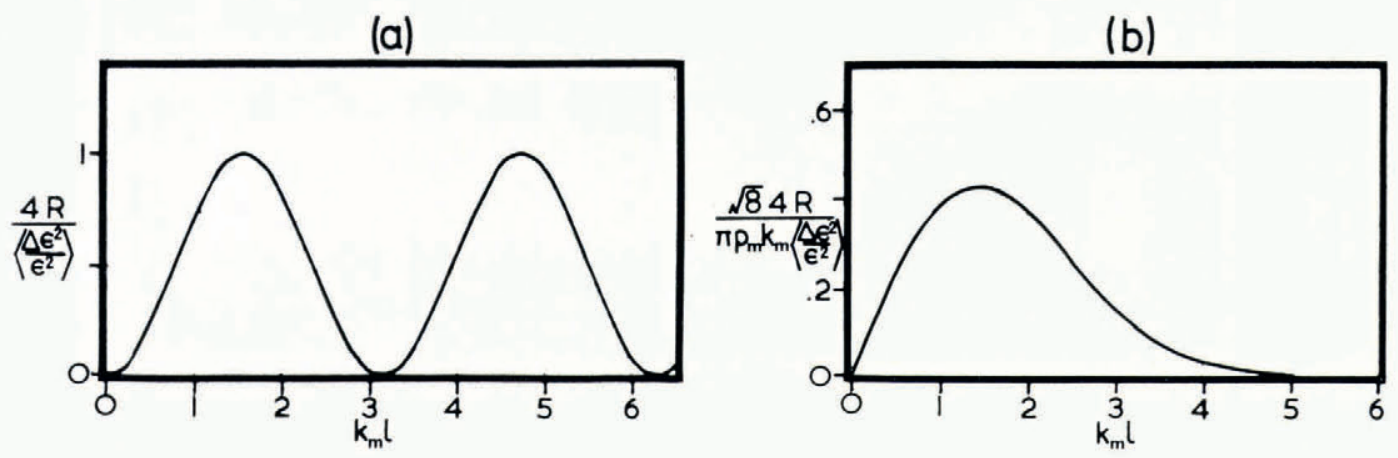

Fig. 5. The variation of the reflection coefficient $R$ with layer thickness $l$ for $(a)$ a single layer and $(b)$ multiple random layers. 
thickness of the ice and $L$ as the initial thickness of a layer at the surface, we can see that if $L<\lambda_{\mathrm{m}} / 2 \pi \sqrt{ } 2$, from Equation ( 17 ) for multiple layers

$$
R=\frac{\pi^{3}}{\sqrt{ } 8} \frac{p_{\mathrm{m}}}{\lambda_{\mathrm{m}^{2}}}\left\langle\frac{\Delta \epsilon^{2}}{\epsilon^{2}}\right\rangle \frac{L h}{H}
$$

and $R$ decreases linearly with depth to zero at the bottom of the ice $(R \propto h)$. If $L>\lambda_{\mathrm{m}} / 2 \pi \sqrt{ } 2$

$$
R=\frac{\pi^{3}}{\sqrt{ } 8} \frac{p_{\mathrm{m}}}{\lambda_{\mathrm{m}^{2}}}\left\langle\frac{\Delta \epsilon^{2}}{\epsilon^{2}}\right\rangle \frac{L h}{H} \exp -\left[k_{\mathrm{m}^{2}} \frac{l^{2} h^{2}}{H^{2}}\right],
$$

so $R$ is very small near the surface of the ice, but increases to a maximum at $h=H \lambda_{\mathrm{m}} / 2 \sqrt{ }{ }_{2} L \pi$ and below this $R$ decreases linearly to zero at the bottom of the ice. For a single thin layer, Equation (7) becomes

and $R \propto h^{2}$.

$$
R=\frac{\pi^{2}}{\lambda_{\mathrm{m}^{2}}}\left(\frac{\Delta \epsilon}{\epsilon_{\mathrm{I}}}\right)^{2} \frac{L^{2} h^{2}}{H^{2}}
$$

It is usually found (Figs. 2 and 3 ) that reflections from layers are not detected in the bottom quarter or so of the total ice depth (depending on recorder sensitivity). This is partly because of the inverse square law, and the possible reduction of $R$ with depth, but mainly due to the increased absorption as the temperature of the ice rises towards the bottom.

It is possible to measure the echo strength of layers as a function of depth from the A-scope display, but generally it is difficult to separate the effects of the variation of absorption and reflection coefficient. For simplicity, let us assume that throughout the depths of interest the reflection coefficient $R$ and the temperature and attenuation per unit path length $\alpha$ are constants. The power received $P_{\mathrm{R}}$ from a plane of reflection coefficient $R$ allowing for refraction and absorption is

$$
\left\{\frac{P_{\mathrm{R}}}{P_{\mathrm{T}}}\right\}=\left\{G^{2}\right\}+20 \log _{10}\left[\frac{\lambda}{8 \pi\left(z_{\mathrm{a}}+z / n\right)}\right]+\{R\}-2 \alpha z
$$

where curly brackets denote the value in decibels and $P_{\mathrm{T}}, G, z_{\mathrm{a}}, z, \lambda, n$ are transmitted power, antenna gain, terrain clearance, depth of the reflector in ice, radio wavelength in air, and mean refractive index of ice $(=1.78)$ respectively. The ordinate of the A-scope display may be calibrated in decibels as in Figure 6 by connecting known attenuators between transmitter

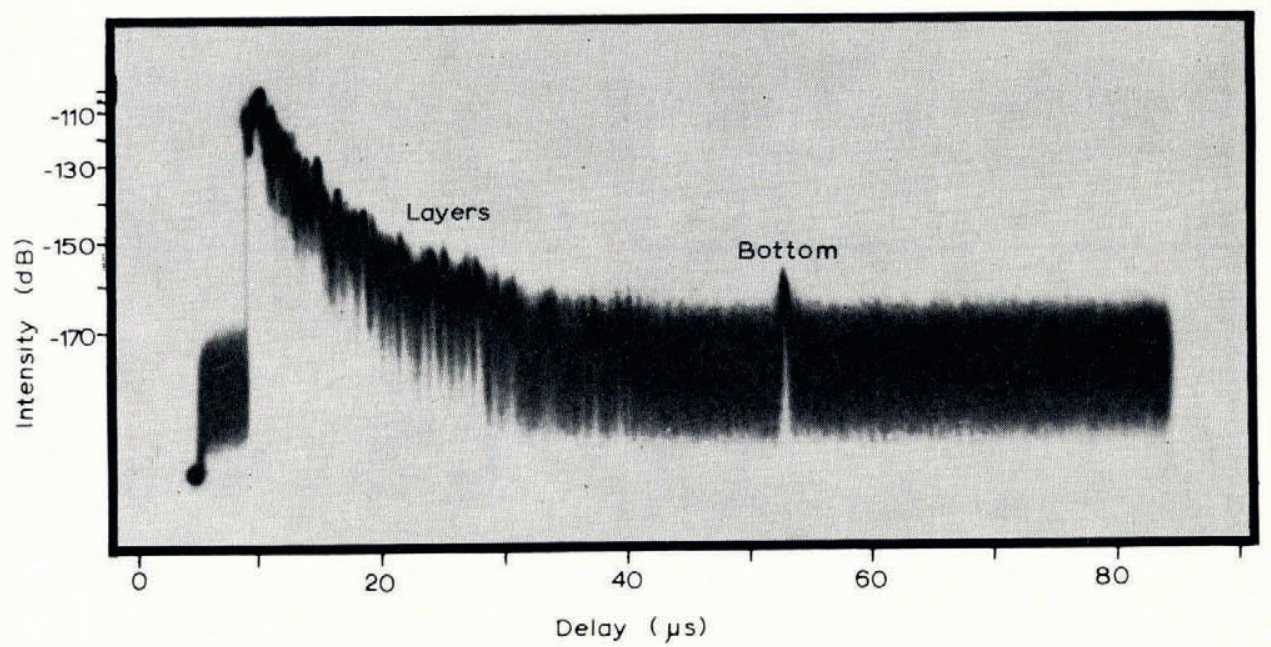

Fig. 6. A typical A-scope display showing layer echoes. The intensity scale is $P_{\mathrm{R}} / P_{\mathrm{T}}$ in $d B$. 
and receiver. It is important to compare A- and Z-scope displays to check that the echoes in question are layer echoes and not large-angle scatter from the ice-air interface. Since the echo strength may fluctuate by io $\mathrm{dB}$ or so over depths of the order of a pulse length, all measurements refer to local power maxima. The reflection coefficient and attenuation per unit length were calculated from the I97 I data (when the carrier frequency was $60 \mathrm{MHz}$ ) by fitting a straight line to the local power maxima between $2000 \mathrm{~m}$ and $3000 \mathrm{~m}$ ice depth and using a linear approximation for the logarithmic term in Equation (23). Table I shows

TABle I. Reflection COEFficients AND ATtenUAtion FOR SITES SHOWN IN FIGURE I

\begin{tabular}{|c|c|c|c|}
\hline & Absorption per unit path & Reflection c & oefficient $R$ \\
\hline Site & $\begin{array}{l}\text { length } \propto \\
\mathrm{dB} / \mathrm{km}\end{array}$ & $\begin{array}{l}\text { Long pulse } \\
\mathrm{dB}\end{array}$ & $\begin{array}{l}\text { Short pulse } \\
\text { dB }\end{array}$ \\
\hline $\mathbf{I}$ & $7 \cdot 0 \underset{(2)}{ \pm} 0.5$ & $-60 \pm 1$ & - \\
\hline 2 & $8.0 \pm \frac{1}{(4)}$ & $-64 \pm I$ & - \\
\hline 3 & $5 \cdot 9 \underset{(9)}{ \pm} 0.7$ & $\begin{array}{c}-64 \cdot 7 \pm 2 \\
(9)\end{array}$ & - \\
\hline 4 & $4 \cdot \frac{0}{(3)} 0.5$ & $\begin{array}{c}-70.6 \pm 4 \\
(3)\end{array}$ & - \\
\hline 5 & $5 \cdot 8 \pm 1$ & $\begin{array}{c}-70.7 \\
(3)\end{array}$ & - \\
\hline 6 & 3.0 & $\begin{array}{r}-82 \\
(1)\end{array}$ & - \\
\hline 7 & $\begin{array}{l}3.2 \pm 0.7 \\
(\text { IO) }\end{array}$ & $\begin{array}{c}-74.8 \\
(6)\end{array}$ & $\begin{array}{c}-8 \mathrm{I} \pm 2 \\
(4)\end{array}$ \\
\hline 8 & $3.7 \pm \frac{7}{(2)} 0.5$ & $\begin{array}{r}-71 \\
(1)\end{array}$ & $\begin{array}{r}-79 \\
(\mathrm{I})\end{array}$ \\
\hline 9 & $3.0 \underset{(2)}{(2)} 0.5$ & -75 & $\begin{array}{r}-79 \\
(\mathrm{I})\end{array}$ \\
\hline IO & $3 \cdot 9 \underset{(5)}{ \pm} 0.5$ & -71 & $\begin{array}{c}-83 \pm I \\
(2)\end{array}$ \\
\hline I I & $2.8 \underset{(2)}{ \pm} 0.2$ & -80 & $\begin{array}{r}-80 \\
(1)\end{array}$ \\
\hline 12 & $3.0 \div \frac{1}{(3)} 0.5$ & $-80, \pm 3$ & $\begin{array}{r}-79 \\
(\mathrm{I})\end{array}$ \\
\hline 13 & $3.5 \underset{(2)}{ \pm} 0.5$ & $-75_{(2)} \pm \mathrm{I}$ & - \\
\hline
\end{tabular}

The numbers in parentheses show the number of points averaged.

the reflection coefficients and attenuation per kilometre for the numbered sites shown in Figure I. Each value is the average of a number of measurements (shown in brackets in each case) from different A-scope displays taken within $70 \mathrm{~km}$ of the particular site. Whenever possible, errors have been estimated from the spread of the measurements at each site. The values of attenuation per kilometre correspond to ice temperatures of $-40^{\circ} \mathrm{C}$ to $-50^{\circ} \mathrm{C}$ (according to Westphal's measurements (Robin and others, 1969), and this agrees with the surface temperatures on the Antarctic Plateau. The order of magnitude of the reflection coefficients is the same as that measured by Robin and others (1969) for layers in Greenland (with an assumed temperature profile). During the $197 \mathrm{I}$ season two transmitter pulse lengths were available, and the values of reflection coefficient obtained with the long transmitter pulse ( $\mathrm{I} \mu \mathrm{s}$ ) are on average about $5 \mathrm{~dB}$ higher than those obtained with the short pulse (250 ns) at the same site. From Equation (6) the strength of reflections from isolated layers should not show any dependence on pulse length, but the strength of echoes from multiple layers (cases 2 and 3 ) should be proportional to the pulse length as shown in Equation (1 7), and an increase of pulse length from 250 ns to $\mathrm{I} \mu \mathrm{s}$ would result in a $6 \mathrm{~dB}$ increase in echo strength. Thus the 
results of Table I suggest that multiple layering is important in these examples. This conclusion is supported by the fact that there is sometimes a small random vertical displacement of some of the layers on changing pulse length as seen in Figure 7.

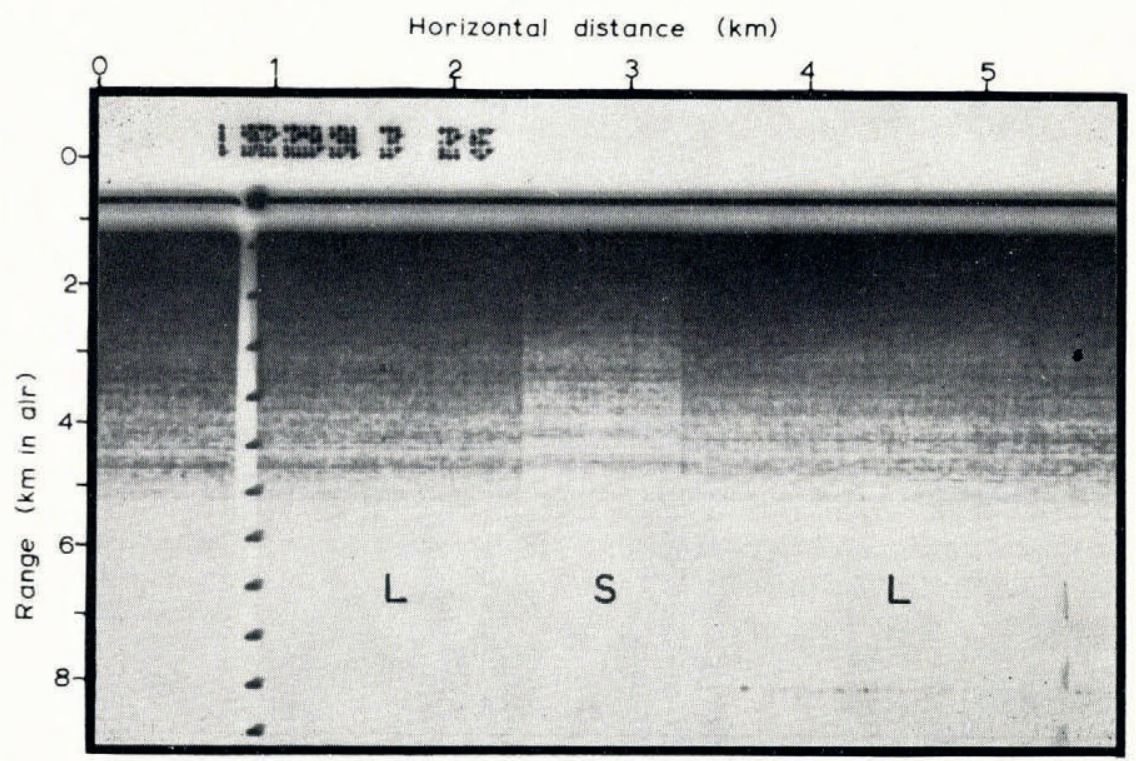

Fig. 7. Radio echo record showing a slight change in position of some of the layer echoes on alteration of pulse length; $L \equiv I \mu$, $S \equiv 250$ ns.

Of the three cases considered, probably the third is the most likely, because climatic variations will produce random changes of dielectric constant with depth usually on a scale much smaller than a pulse length, but their scale may change as the ice is plastically deformed at depth. Because of these variations there will be some background reflection whose magnitude varies on a vertical scale of about one pulse length but whose average strength is given by Equation (17). Superimposed on this background there may be reflections from a large (random) discontinuity in dielectric constant sufficient to cause a discrete echo whose magnitude, given by Equation (6), is much greater than the background. However, it may be seen that the latter situation is highly unlikely with equipment having S.P.R.I. Mk II or Mk IV parameters. From Equation ( 17 ) the reflection coefficient of closely spaced multiple layers $\left(k_{\mathrm{m}} l \ll \mathrm{I}\right)$ is proportional to $\left(p_{\mathrm{m}} l / \lambda_{\mathrm{m}}^{2}\right)\left\langle\Delta \epsilon^{2} / \epsilon^{2}\right\rangle$ and for a single layer of thickness $l^{\prime}$ the reflection coefficient is proportional to $l^{\prime 2} \Delta \epsilon(z)^{2} / \lambda_{\mathrm{m}^{2}} \epsilon^{2}$. Thus, if the discrete echo is to be stronger than the background we must have $\left.\Delta \epsilon(z)^{2} / \epsilon^{2}\right\rangle\left(p_{\mathrm{m}} l / l^{\prime 2}\right)\left\langle\Delta \epsilon^{2} / \epsilon^{2}\right\rangle$, and since $l$ and $l^{\prime}$ may be of the order of $\mathrm{I} \mathrm{cm}, p_{\mathrm{m}} l / l^{\prime 2} \approx 2$ ooo. So the single layer must have a dielectric constant such that $\Delta \epsilon^{2}$ is 2 ooo times greater than the mean square value of $\Delta \epsilon$ over a depth of about a pulse length, and this is extremely unlikely.

\section{HorizontAl GORRELATION OF LAYER EGHOES}

Up till now we have assumed that the variation of $\Delta \epsilon$ constituting the layers is a function of $z$ only, but this clearly cannot be absolutely true because the echoes often fade as the observer moves (Fig. 8). The fading pattern on a particular layer echo may have been caused by (a) the surface roughness or variations in reflectivity of the layer itself, (b) differences in path length in passing through the layered medium above the layer (c) the roughness of the 


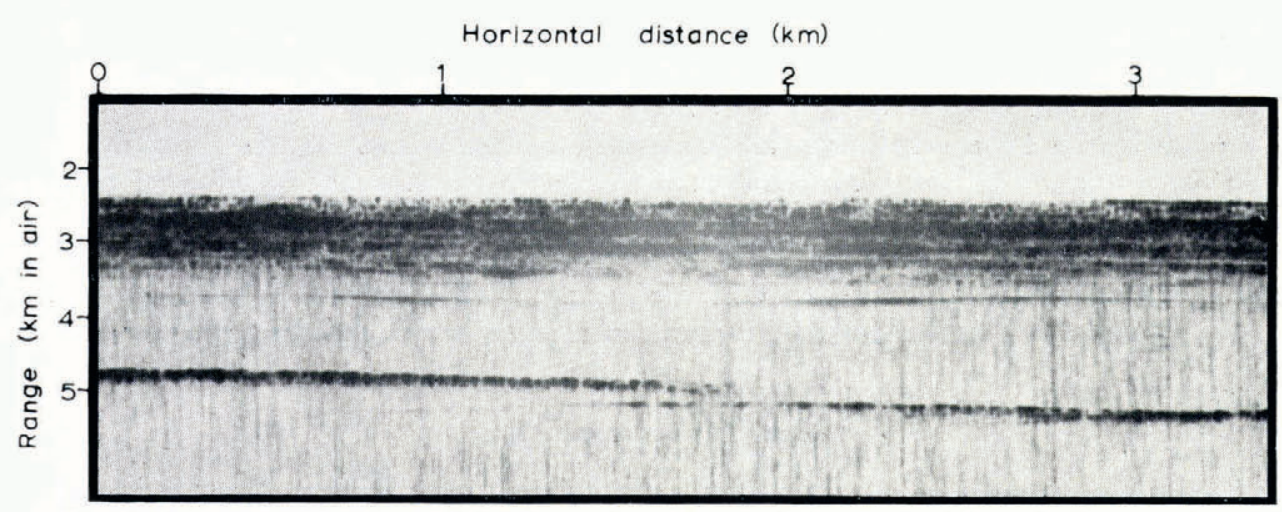

Fig. 8. Radio echo record showing fading layer echoes where the fading rates at different depths are unrelated.

top of the ice (this is discussed in more detail in Harrison (1971, unpublished, p. I08). In cases (b) and (c) we might expect some degree of correlation between fading patterns from layers at different depths whereas in case (a) the fading patterns are likely to be uncorrelated. It is often found that the fading rate of layer echoes varies randomly with depth as shown in Figure 8 suggesting that each fading pattern is genuinely associated with its own layer. It is not clear whether the fading is due to changes in reflection coefficient caused by variation of $\Delta \epsilon$ or $l$, or to the roughness or waviness of the layers.

A more detailed treatment is possible starting from Equation (3) and deriving fading lengths, but it is difficult to describe statistically a medium which may at any one depth be correlated over many kilometres and yet cause fading on a scale of tens or hundreds of metres. Physically, however, it is clear that layers associated with climatic changes (annual layers, for example) may be correlated over extremely large distances, while locally they may have been perturbed during formation at the surface by winds. Sastrugi may have amplitudes larger than the annual accumulation $(\approx 5 \mathrm{~cm}$ on the inland ice sheet (Chorlton and Lister 1970) ) and this results in false bedding (Koerner, 1964), so we might expect there to be local variations of $\Delta \epsilon$ causing fading but not destroying the large scale layered structure. It is likely

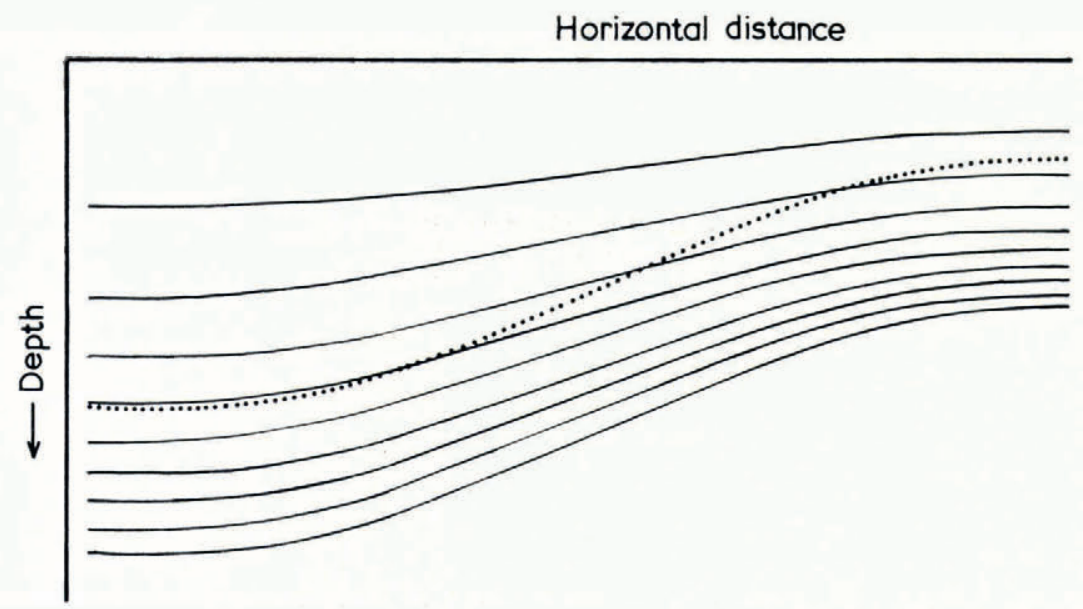

Fig. 9. Diagram showing a lize of constant reflection coefficient (constant layer thickness) (dotted line) as the layered ice passes over mountainous bedrock. 
that annual layers act as near specular reflectors since their vertical spacing is usually much less than a radio wavelength, and so the fading length is expected to be the same as the characteristic spacing of local variations in $\Delta \epsilon$.

If the layered ice flows gently over an obstacle in the bedrock so that the ice thickness $H$ is reduced as in Figure 9, then, assuming uniform vertical strain, the spacing of the layers reduces in proportion to $H$, and $L \propto H$. For gentle undulations, using Equation (I9), $l=L h / H$ where $L / H$ is independent of position, and so a line of constant reflection coefficient stays at a constant height above bedrock as in Figure 9. This effect is seen in Figure ro where the depth to which echoes are detected remains at a roughly constant height above bedrock.

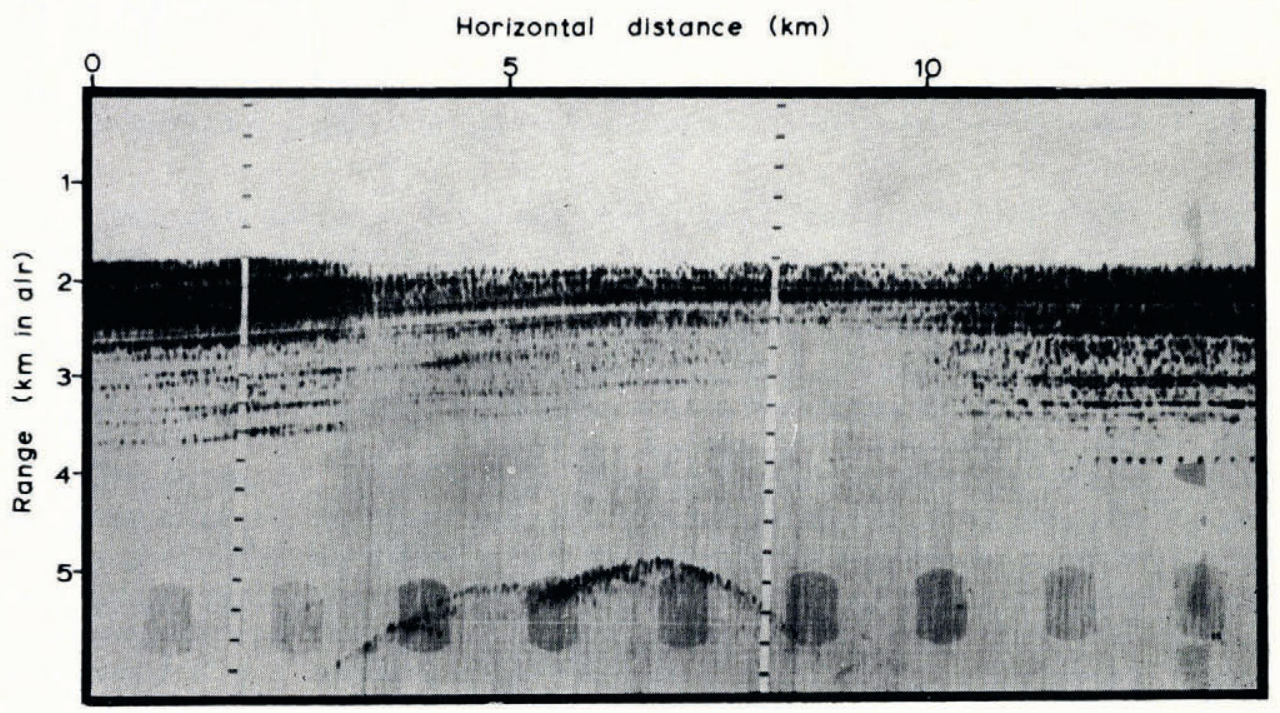

(a)

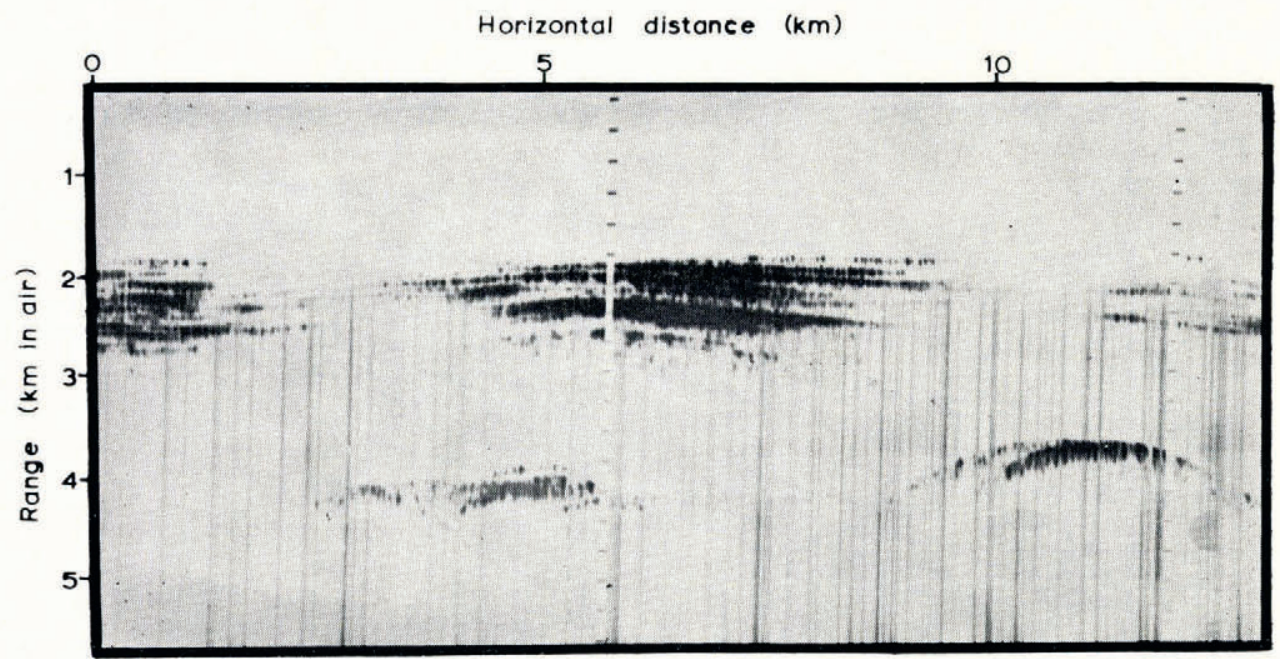

(b)

Fig. 10. Radio echo records showing weakening of the layer echoes over mountainous bedrock. 
Another, and possibly more important, cause of weak echoes in this situation may be increased absorption in the ice resulting from frictional heating during deformation (Paterson, 1969, p. $\left.{ }_{7} 78\right)$.

\section{Possible gauses of LAyering}

The differences in dielectric constant between one layer and another may be caused by differences in impurities, in orientation of anisotropic material, or density variations.

According to Schytt (1958, p. 122) the transformation from firn (bubbly ice) to clear ice may be fitted to an exponential variation of density $d$ with depth $z$

$$
d(z)=d_{0}-d_{\mathrm{I}} \exp \left(-\frac{z}{z_{\mathrm{c}}}\right)
$$

where (on the ice shelf at Maudheim) $d_{0}=0.9^{2} \mathrm{Mg} \mathrm{m}^{-3}, d_{\mathrm{I}}=0.45 \mathrm{Mg} \mathrm{m}^{-3}, z_{\mathrm{c}}=3^{8} \mathrm{~m}$. As the density of clear ice is $d_{0}$, Equation (24) gives us an upper limit for the density difference between bubbly and clear ice as a function of depth. Using the empirical relationship from Robin and others (1969),

$$
\sqrt{ } \epsilon=n=\mathrm{I}+0.85 d,
$$

we see that for layers consisting of alternately bubbly and clear ice

$$
\left\langle\Delta \epsilon^{2} / \epsilon^{2}\right\rangle \lesssim\left[2 \times 0.85 d_{\mathrm{I}} / \sqrt{ } \epsilon_{\mathrm{I}} \exp \left(-z / z_{\mathrm{c}}\right)\right]^{2},
$$

and so the reflection coefficient of the layers as a function of depth is from Equation (20) (where $l=h L / H$ )

$$
R(z)=\frac{\pi^{3}}{\sqrt{ } 8} \frac{p_{\mathrm{m}}}{\lambda_{\mathrm{m}^{2}}} \frac{L(H-z)}{H} \frac{\left(2 \times 0.85 d_{\mathrm{I}}\right)^{2} \exp \left(-2 z / z_{\mathrm{c}}\right)}{\epsilon_{\mathrm{I}}} .
$$

Putting $p_{\mathrm{m}}=22 \mathrm{~m}, \lambda_{\mathrm{m}}=4.8 \mathrm{~m}, L=5 \mathrm{~cm}, H=3000 \mathrm{~m}$, the reflection coefficient at $z=200 \mathrm{~m}$ is $R \approx 6 \times 10^{-5}$ or at $z=400 \mathrm{~m}, R \approx 3 \times \mathrm{IO}^{-11}$. These density variations cannot account for the observed strength of the layer reflections at depths of 1 ooo- $3000 \mathrm{~m}$.

Several dirt bands (probably volcanic ash) in the Byrd bore hole have been reported by Gow (1970) at depths between I 300 and I $700 \mathrm{~m}$. The bands are about $0.5 \mathrm{~mm}$ thick but they are always associated with a layer of refrozen melt water about $10 \mathrm{~mm}$ thick. Treating the dirt band as a dilute mixture of rock particles $\left(\epsilon_{1}\right)$ in ice $\left(\epsilon_{2}\right)$ we have $\Delta \epsilon=3^{m}\left(\epsilon_{1}-\epsilon_{2}\right)$ / $\left(\epsilon_{\mathrm{I}}+2 \epsilon_{2}\right)$ (Stratton, I $94 \mathrm{I}$, p. 572) where $m$ is the fraction by volume of rock particles, and assuming $\epsilon \approx 5$ and $\epsilon_{2}=3.17$ we have $\Delta \epsilon=0.48 \mathrm{~m}$. So, from Equation (7) the reflection coefficient with $m=10 \%$ for a single layer $0.5 \mathrm{~mm}$ thick is $R=-106 \mathrm{~dB}$, and for a layer I $\mathrm{cm}$ thick $R=-8 \mathrm{o} \mathrm{dB}$. Thus, ash layers may be responsible for some of the deeper echoes but as a rule they are too weak and rare to explain them all.

Since ice is structurally anisotropic its radio-frequency dielectric constant might be expected to be anisotropic and layers containing crystals alternately in a preferred and random orientation might give detectable reflections. No laboratory measurements of dielectric anisotropy in ice have been made, but the standard deviation of measurements of the mean dielectric constant is of order o.or (Paren, private communication), so the difference due to anisotropy is probably less than this.

Some light is thrown on the question of the existence of radio-frequency dielectric anisotropy in ice by an experiment of Jiracek's (unpublished, p. 9o). Jiracek made measurements at several sites of the polarization of radio echoes and he concluded that his results could only be explained by the anisotropy of ice. The purpose of the experiment was to investigate the changes of polarization on reflection at bedrock, which depend on angle of incidence and reflection and the roughness of the reflecting surface (Beckmann and Spizzichino, 1963, p. I 52). He used a spaced transmitter and receiver with a (linearly polarized) transmitting antenna in a fixed but arbitrary orientation in the horizontal plane and with the receiving 
antenna at a variable angle to the transmitting antenna. According to the theoretical argument of Beckmann and Spizzichino ( 1963 , p. I63), with transmitter and receiver close together there should be no depolarization on reflection (a rotation of $180^{\circ}$ is not detectable). However, Jiracek found that the received signal was plane polarized but rotated through $90^{\circ}$ at Amundsen-Scott station (South Pole), and elliptically (almost circularly) polarized on the Skelton Glacier. A small rotation of polarization could be explained by Faraday rotation in the Earth's magnetic field (rotation due to natural optical activity, if present, would cancel out on the return journey), but the simplest explanation of both Jiracek's observations is that the polarization is altered by birefringence in the ice.

Suppose we have a birefringent medium in which the maximum refractive index $n_{x}$ is along the $x$-axis and the minimum $n_{y}$ is along the $y$-axis (in a Cartesian coordinate system), and the difference $\Delta n=n_{x}-n_{y}$ is much less than $n_{x}$. A plane, linearly polarized wave propagating in the $z$ direction has components of electric field $E_{x}, E_{y}$ in the $x, y$ directions respectively, and since $n_{y}$ is smaller than $n_{x}$ the component $E_{y}$ will travel slightly faster than $E_{x}$, setting up a phase difference between $E_{y}$ and $E_{x}$ given by

$$
\phi=k_{\mathrm{m}} \Delta n z_{0}
$$

where $z_{0}$ is the thickness of the medium.

In general, the vector addition of $E_{x}$ and $E_{y}$ results in an elliptically polarized wave, and the total phase difference between $E_{x}$ and $E_{y}$ in passing through a thickness $z_{0}$ of the medium and reflecting back to the origin is twice the value given in Equation (27).

Although this simple theory cannot cope with a medium in which the direction of the maximum refractive index varies with depth, it shows that a very small value of $\Delta n$ could account for the observations. Substituting $z_{0}=3000 \mathrm{~m}, k_{\mathrm{m}}=2 \pi / 5 \mathrm{~m}^{-1}$ in Equation (27) a phase change of $\phi=\pi / 2$ (causing elliptical polarization, in general) would be given by $\Delta n \approx 2 \times \mathrm{IO}^{-4}$. Jiracek's results suggest that, on average over the depth of ice, the maximum refractive index in the horizontal plane must lie preferentially in some particular direction, and since ice crystals are uniaxial the $c$-axes must be, on average, inclined away from the vertical at some particular azimuth. The importance of Jiracek's work in the context of this paper, however, lies in simply demonstrating the existence of radio-frequency dielectric anisotropy, and it is clear that there is no further evidence for the existence of layers of differing crystal orientation.

\section{Conclusion}

The lack of direct evidence for the existence of variation of $\epsilon$ with depth is probably due to the smallness of $\Delta \epsilon$. It is clear that measurement of $\epsilon$ to $\pm \mathrm{IO}^{-4}$ in core samples is difficult in the laboratory, and possibly a more useful approach would be to study the dependence of the layer echoes at a given site on radio wavelength, pulse length and depth.

The dependence of the effective reflection coefficient of layers on radio-frequency may be measured (provided absorption effects can be eliminated) and compared with Equation (6) or ( 17$)$. $l$ and $\left\langle\Delta \epsilon^{2}\right\rangle$ may be separated and evaluated by finding the frequency at which $R$ is a maximum when $l=\lambda_{\mathrm{m}} / 2 \pi$ for multiple layers, Equation ( 17 ), or $l=\lambda_{\mathrm{m}} / 4$ for an isolated layer, Equation (6). Also, by taking the Fourier transform of both sides of Equation (14) we have

$$
\rho(\zeta)=\frac{8 \sqrt{ } 2}{\pi^{\frac{3}{2}} p_{\mathrm{m}}\left\langle\left(\frac{\Delta \epsilon}{\epsilon}\right)^{2}\right\rangle} \int_{-\infty}^{\infty} \frac{R\left(k_{\mathrm{m}}\right)}{k_{\mathrm{m}}^{2}} \exp \left(2 \mathrm{i} k_{\mathrm{m}} \zeta\right) \mathrm{d} k_{\mathrm{m}},
$$

and knowing the mean reflection coefficient as a function of frequency $R\left(k_{\mathrm{m}}\right)$ we may calculate the shape of the autocorrelation function of dielectric constant $\rho(\zeta)$ (which has so far been 
assumed to be a Gaussian, Equation (9)). Similarly, the dependence of the reflection coefficient on pulse length at any one site and the possible variation in position of the layer echoes with pulse length and radio frequency might determine more conclusively than Table I whether the layers are multiple or single.

The changes of $\Delta \epsilon$ with depth responsible for the layers is probably best explained at present by variations of ice crystal orientation since this is the only mechanism capable of giving practically continuous variations at large depths. However, density variations in the top few hundred metres and the refrozen melt associated with ash layers may still account for some of the observations.

MS. received 3 November 1972

\section{REFERENCES}

Beckmann, P., and Spizzichino, A. 1963. The scattering of electromagnetic waves from a rough surface. Oxford, Pergamon Press. (International Series of Monographs on Electromagnetic Waves, Vol. 4.)

Booker, H. G., and Gordon, W. E. 1950. A theory of radio scattering in the troposphere. Proceedings of the Institute of Radio Engineers, Vol. 38, No. 4, p. 40I-12.

Chorlton, J. C., and Lister, H. r970. Snow accumulation over Antarctica. [Union Géodésique et Géophysique

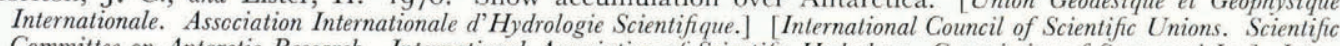
Committee on Antarctic Research. International Association of Scientific Hydrolog): Commission of Snow and Ice.] International Symposium on Antarclic Glaciological Exploration (ISAGE), Hanover, New Hampshire, U.S.A., 3-7 September 1968 , p. $254-63$

Evans, S., and Smith, B. M. E. 1969. A radio echo equipment for depth sounding in polar ice sheets. Journal of Scientific Instruments (Journal of Physics, E), Ser. 2, Vol. 2, No. 2, p. 131-36.

Evans, S., and others. 1972. Radic-echo sounding in Antarctica, 1971-72, by S. Evans, D. J. Drewry and G. de Q. Robin. Polar Record, Vol. 16, No. 101, p. 207-12.

Gow, A. J. 1970. Preliminary results of studies of ice cores from the $2164 \mathrm{~m}$ deep drill hole, Byrd station, Antarctica. [Union Géodésique et Géophysique Internationale. Association Internationale d'Hydrologie Scientifique.] [International Council of Scientific Unions. Scientific Committee on Antarctic Research. International Association of Scientific Hydrology. Commission of Snow and Ice.] International Symposium on Antarctic Glaciological Exploration (ISAGE), Hanover, New Hampshire, U.S.A., 3-7 September 1968, p. 78-90.

Gudmandsen, P. r970. Notes on radar sounding of the Greenland ice sheet. (In Gudmandsen, P., ed. Proceedings of the international meeting on radioglaciology, Lyngby, May 1970. Lyngby, Technical University of Denmark, Laboratory of Electromagnetic Theory, p. 124-33.)

Harrison, C. H. 1970. Reconstruction of subglacial relief from radio-echo sounding records. Geophysics, Vol. 35, No. 6, p. $1099^{-115}$.

Harrison, C. H. 197I. Radio-echo sounding: focusing effects in wavy strata. Geophysical Journal of the Royal Astronomical Society, Vol. 24, No. 4, p. 383-40o.

Harrison, C. H. Unpublished. Radio propagation effects in glaciers. [Ph.D. thesis, University of Cambridge, 1972.]

Jiracek, G. R. Unpublished. Radio sounding of Antarctic ice. [M.Sc. thesis, University of Wisconsin, I965.]

Koerner, R. M. I964. Firn stratigraphy studies on the Byrd-Whitmore Mountains traverse, 1962-63. (In Mellor, M., ed. Antarctic snow and ice studies. Washington, D.C., American Geophysical Union, p. 219-36. (Antarctic Research Series, Vol. 2.))

Nye, J. F. I963. Correction factor for accumulation measured by the thickness of the annual layers in an ice sheet. Journal of Glaciology, Vol. 4 , No. 36 , p. $785-88$.

Paterson, W. S. B. 1969 . The physics of glaciers. Oxford, Pergamon Press. (The Commonwealth and International Library. Geophysics Division.)

Ratcliffe, J. A. 1956 . Some aspects of diffraction theory and their application to the ionosphere. Reports on Progress in Physics, Vol. 19, p. 188-267.

Robin, G. de Q., and others. Ig69. Interpretation of radio echo sounding in polar ice sheets, by G. de Q. Robin, S. Evans and J. T. Bailey. Philosophical Transactions of the Royal Society of London, Ser. A, Vol. 265, No. I I66, p. $437-505$.

Robin, G. de Q., and others. I970. Radio echo exploration of the Antarctic ice sheet, by G. de Q. Robin, C. W. M. Swithinbank and B. M. E. Smith. [Union Géodésique et Géophysique Internationale. Association Internationale d'Hydrologie Scientifique.] [International Council of Scientific Unions. Scientific Committee on Antarctic Research. International Association of Scientific Hydrology. Commission of Snow and Ice.] International Symposium on Antarctic Glaciological Exploration (ISAGE), Hanover, New Hampshire, U.S.A., 3-7 September 1968, p. 97-1 15.

Schytt, V. 1958. Glaciology. II. The inner structure of the ice shelf at Maudheim as shown by core drilling. Norwegian-British-Swedish Antarctic Expedition, 1949-52. Scientific Results, Vol. 4, C.

Stratton, J. A. 1941. Electromagnetic theory. New York, McGraw-Hill Book Co., Inc. 